\title{
Study on the Application of Music Psychology in Adolescent Mental Education
}

\author{
Bin $\mathrm{Li}^{1}$ \\ ${ }^{1}$ Luohe Medical College, Luohe, Henan, 462000 \\ ${ }^{a}$ email
}

Keywords: Music Psychology; School Students; Mental Health

\begin{abstract}
Adolescent school students, they are experiencing life and learning all sorts of confusion and perplexity, subjects learn to deal with growing psychological problems encountered in the process, learn the desired growth experience, so in the knowledge of psychology into their own internal feelings, which for their future growth would have a negligible effect. And the music, such a special media, music psychology, this emerging discipline in the students' psychological health education plays an important role, and play a special and irreplaceable role to explore the subject in secondary mental health education in use, will help seek counseling right for middle school students, allowing them to be more physically and mentally healthy growth.
\end{abstract}

\section{Introduction}

Due to rapid social change, the pace of life, increasing competition, a substantial increase in high school students facing problems and puzzles, in middle school, students' psychological problems have become more serious, and the level of psychological quality directly affects the quality of the development and direction of development of other qualities. Therefore, attention to psychological health of middle school students, to enhance their ability to adapt to society, has become an important task for the 21st century school education. Music psychology as a new cross-disciplinary, clinical practice has been made in the medical and psychological counseling of a significant effect, but it went into basic mental health education is also relatively rare, the following music psychology at the Mental Health Education practice some discussion.

\section{The Music And Mental Health Understanding}

Music is a natural sound. It is the tone with a rhythm, rhythm, melody and speed, audio and other features. Tone from the natural sound emitted to the formation of music from a single tone to a complete piece of music, which has to go through a series of processing, transformation and cultural decoration process. The nature beautiful sound, sound, thunder rain, wind, springs gurgling sound of water, empty mountain birds, cicadas, frogs and other sounds can also be likened to natural music. Whether natural music, or processed by the human spirit from the music, not only for people who can bring some kind of spiritual enjoyment, but also with human health and longevity also occur close relationship. About music on physical and mental health of the relationship, the ancients as early as thousands of years ago has a unique perspective, "Music" in the records, the gentleman said: Andean ritual can not be required to go to the body, causing the heart to rule the music is easy to Zhi sub understanding of the heart, spontaneously students carry. Yi Zhi is the heart of the child understanding music, music is security, security is a long, long time the heavens, the God. And Plato also mentioned: Music can be "put people into the United States and good education."

Psychology is the psychology of music theory, using methods of experimental psychology, research and interpretation by the original people to advanced musical experience and musical acts. Its research is very extensive, such as the physical characteristics of sound in the human auditory reflection of musical memory, musical imagination, musical talent and musical skills training and psychological state of music and so on. It is because of the relationship between music psychology with psychological methods and theoretical study of music and musical works of human influence, as well as a variety of psychological phenomena between music and people, making it the status of 
science in music therapy is no trivial matter. Music therapy began in the 1950s, it psychological treatment based on unique physiological and psychological effects with music, with the joint participation of the music therapist, through a variety of musical acts specifically set up so that Qiu Zhizhe experience music experience, to eliminate the psychological barriers or enhance physical and mental health purposes.

Music Taste is a mental activity of people, and the people hearing it directly. In the musical aesthetic process, the specific sound of musical art as a suitable stimulus effect on the human auditory analyzer cause hearing, thus acting on the human body and mind, to produce aesthetic pleasure. --- This is a stimulus response pattern. In principle psychotherapy profession of music therapy, although different explanation, but it certainly has a therapeutic effect reached a consensus. Swedish School of Music Psychotherapy founder Pontwick carefully studied the psychological resonance theory, he thinks, by the sound of music and sound system reflects some primitive form of spiritual life, gentle and smooth music people comfort, and loud, cheerful the music is people excited, excited. Studies have shown that the right music can eliminate the bad experiences of the patient, but also to expand areas of feeling and experience it can enjoy, but also to the structure of thinking in the process of listening to music can be improved. So most patients can be music psychotherapy based on the principles of these difficult to understand. The parent of American Music Therapy Gaston said: "The power and value of music lies in its non-linguistic connotation." This exchange is a key factor in the characteristics of the music for the clinical treatment, especially when the language of the efforts fizzle Music can help establish a good doctor-patient relationship, and this relationship is the treatment of the basic driving force for success. In addition, the music is present in a real time and by the physical structure (air molecules vibrate) formed there. This reality can be heard, felt, and measured out with drawings and symbols, so music can be an effective medium to help patients who retreat from reality and society out of the back to the real world, establish contact with the outside real world.

\section{Music Psychology in Secondary Education in the Use of Mental Health}

Adolescence is a critical period of physical and mental development of students, but also the physical and mental development of the students during the mutation. Both in terms of their physiological or psychological aspects there are many contradictions and confusion, according to the principles of the psychology of music, the unique charm of music

And Beneficial penetration in middle school mental health education can better help them successfully through this period, the maintenance and promotion of mental health, prevention of mental illness. Than the traditional mental health education, music, counseling and treatment of other media can not be compared with a unique advantage.

In China, currently the most commonly used high school, is the most widely used psychological health education classroom teaching, according to the students' psychological development characteristics, life experiences from existing student starting to arouse their cognition, emotion and behavior of a variety of experiences The system can help students regulate emotions, sunshine state of mind, self-understanding, develop their potential. It is an effective mental health education model. If you can focus on the psychological characteristics of high school students, the use of the special nature of the music media, the appropriate use of music in secondary mental health classroom teaching to counseling and assistance, will be able to achieve an unexpected effect. The following is one of Case in point:

Topic: hold up your dreams

The goal: a dream inspiring experience; 2 establish self-growth objectives; 3 learning self management by objectives, design dreams come true ways and methods. Design ideas:

The name of the story. Share stories, "the name of the story," import the theme. I like my own name, it is: — but if possible, I want my name to: $\longrightarrow$, because it represents the meaning ........... exchange. Group exchanges, and then focus on the class exchange. To emotionally charged Tell Me, in the whole process, teachers should certainly timely story contains student name "dream" beauty and "dream" a positive impact on self-growth. Summary: ordinary 
name, contains a great dream. Each of us has their own dream, the dream is to grow our way a Venus, urging them forward, encouraging people to rise up, let us dream of starting today sown the seeds of it.

Under meteor desire. (1) ancient legend, when the meteor across the sky, the promise of our desire can be achieved, if the current positive meteor across the sky, you have time to make their promised five more wishful thinking ...... (2 ) students Make a wish under the meteor. While playing background music, Bandari Orchestra "new morning". (Here the use of soft, dreamy melodies create a meteor atmosphere, as if opening up together in the quiet place in the hustle and bustle of the city, to help students sink in the heart of the inquiry of his own heart, which found that they really want to pursue the real thing and the desire to achieve the purpose of teaching this link. (3) to recover the desire to strip, at random, all the campers exchange, teachers appropriate reviews.

The teacher tells the story exchange. Bandari here still use music as a background, but the volume should be adjusted relatively weak, so as not to affect the students speak better. Indistinct melody to create a subtle atmosphere, better to let the feelings of students receive reminders

Emotional venting, especially some children special family circumstances, the reaction in this atmosphere is more obvious, because usually they do not have a chance, or that they do not know their hearts are hidden some of the pent up feelings and, the equivalent to these children the opportunity to express themselves a heart, relieve their emotions, their mental health has a good impact. (2) panel discussion. (3) teachers and students are summarized: the secret dream come true.

Small days with big dreams, any wind to dry, flowing tears and sweat, I someday have their own day, pioneering life, dreams hold firmly towards goal, you life will certainly be able to find their own blue sky!

All campers singing "Invisible Wings" by the end of this sub-theme activities. Here you select the song inspirational song, because it is not only high school students are familiar with, and more important to use at the end of course, there will be a classroom content for deepening and sublimation

With, opportunities for students to accumulate before a few links in the emotional outbreak, deepen the theme of the lesson, waves, icing on the cake.

Test anxiety is one of the Middle School Students common mental disorders, particularly grade graduate students, many students due to the effect of test anxiety in the exam, college entrance examination is not the proper level, ranging from the examination room can not concentrate, tremble affect writing exam long-term insomnia, severe "halo field", so that the examination can not be carried out. For students' test anxiety, people often use the method of reason, children do ideological work, but the results are often poor. For graduating seniors prevalence of test anxiety problems, especially graduating seniors started music exam anxiety mental-stress group lessons, not only can quickly and effectively eliminate the negative effects due to past failures exam experience brought, but also help students improve their range of anxiety symptoms, by way of group counseling were students of this new activity also feel very fresh, happy to accept.

Middle School Graduates life is full of stress and pressure, and long-term state of anxiety not only cause examination to play mad, and more importantly, will cause a variety of physical and mental diseases, a direct impact on the health of students. The music can act directly on the central nervous system, the hypothalamus and the edge of the brain in charge of emotions, human emotions for two-way adjustment. Relaxation training is a behavioral therapy commonly used technique for stress, anxiety, restlessness, anger and emotional situations can be very useful to help students keep up your spirits, restore strength, fatigue, calm mood. The method is simple, saving time. As long as the high school counseling room equipped with certain audio equipment can be carried out. The main relaxation include: progressive relaxation training, relaxation training imagination, take a deep breath to relax (natural relaxation method).

Insomnia is a common sleep disorder, is one of the most common symptoms of neurasthenia, seriously affect the individual's health. Year of high school graduates because of the forthcoming examinations, psychological pressure, prone to insomnia, obviously very tired, but it is not sleep. Insomnia suffer a student, under some frustration turned to drugs, not only physical injury, but the 
effect is not how good. I choose the right music, alleviate or eliminate their stress, anxiety and irritability, insomnia and other symptoms. And induce sleep to make it go to sleep to music instead of drugs. I would recommend insomnia students each year in prep can listen to some beautiful melody, rhythm crisp, sweet harmonies and classical music light music before going to sleep every day to help them fall asleep. And according to their different personalities and preferences of each choose different types of music to help sleep: In general, depressive personality students should listen to a beautiful melody and smooth, fast-paced, joy melody music, such as: "water", "step by step" "radiant" and so on; and anxiety are quite serious students listen to the appropriate Qingli elegant melody, rhythm slow, leisurely atmosphere, elegant styles of music, such as: "Red Beans", "song of Triumph", "Suzhou line" and so on; emotional impulses, irritability students recommend them to listen to beautiful melodies, quiet and sweet, rhythmic melody of music, such as: "moonlit Night", "song of the Frontier" and so on. Music can regulate the heart rate and breathing, insomnia, relieve stress, forget the troubles sorrow, eliminate anxiety and irritability, mood adjustment, people calm, emotionally stable, relaxed, quiet, thereby improving sleep. Of course, the use of music therapy for insomnia should also pay attention to the time constraints, I recommend that students generally choose the evening before going to bed 2-3 hours, that is going to night classes to go home after the time, if there was not time can be 2 to 3 times a day treatment time is 30 to 60 minutes, not too long, not with a single, so boring. Volume is not too large, comfortable for the degree, should be controlled below 70 decibels, music should be put into the body, seek experience from the music, and also can self humming with music. After listening to the music activities were walking, and the family can talk to some of the interesting, avoid talking about learning and life vexed question. Such music therapy treatment of insomnia generally a month for a course. Generally speaking, the lighter a course of insomnia have a significant effect, the heavier the students will need in the months before the exam began to soon before exams can receive better treatment. .

\section{Music Psychology Prospects in Mental Health Education Prospects}

With the popularity of a new concept of modern education curriculum reform, quality education, lifelong education, the importance of mental health education has become more evident, since work in the education and practice of music combining Mental Health, I increasingly seeing the beauty of music is how to motivate students and evokes a powerful force deep inside a positive force and life. I sighed and said: even with this music so special and powerful! Seen in this light, music psychology as a new cross-disciplinary, not only is the use of medical treatment in certain areas, you can also play its unique effect on the basis of mental health education, the most natural medium to help students answer growing confusion, to appease the youthful soul!

\section{Acknowledgements}

This is the Science and Technology Department of Henan Province Soft Science Research Program Achievements in 2014. Item Number: 142400410239

\section{References}

[1] Gao Tian. Introduction to Music Therapy [M]. World Publishing Company, 2008-05, (1).

[2] David R Shaffer, Zou Hong, such as translation. --- Developmental psychology of children and adolescents (sixth edition) [M]. China Light Industry Press, 2007-01, (2).

[3] Luo Xiaoping, Hong Huang Music Psychology [M]. Shanghai Conservatory of Music Press, 2010-03, (2).

[4] Ji Likang. Annotation music note Translation [M] Beijing: Music Publishing House, 1958-03, (1).

[5] Zhou Shibin. Music Education and Psychology Research Methods [M]. Shanghai Music 
Publishing House, 2006-07, (2).

[6] Yang Zhiliang, Bian Yufang. Psychology (School Mental Health Education Series) [M]. East China Normal University Press, 2008-06, (7).

[7] Xu Xuejun. Juvenile counseling [M]. Hubei People's Publishing House, 1995-03, (1). 